\title{
Differences in peripheral noradrenergic function among actively drinking and abstinent alcohol-dependent individuals.
}

\author{
Ashwin A. Patkar \\ Thomas Jefferson University \\ Charles A. Marsden \\ University of Nottingham Medical School \\ Prakash C. Naik \\ Lyndon Clinic \\ David A. Kendall \\ University of Nottingham Medical School \\ Eollow this and additional works at: https://jdc.jefferson.edu/phbfp \\ Raman Gopalakrishnan

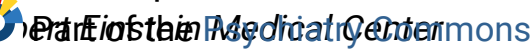 \\ Let us know how access to this document benefits you
}

See next page for additional authors

\section{Recommended Citation}

Patkar, Ashwin A.; Marsden, Charles A.; Naik, Prakash C.; Kendall, David A.; Gopalakrishnan, Raman; Vergare, Michael J.; and Weinstein, Stephen P., "Differences in peripheral noradrenergic function among actively drinking and abstinent alcohol-dependent individuals." (2004).

Department of Psychiatry and Human Behavior Faculty Papers. Paper 46.

https://jdc.jefferson.edu/phbfp/46

This Article is brought to you for free and open access by the Jefferson Digital Commons. The Jefferson Digital Commons is a service of Thomas Jefferson University's Center for Teaching and Learning (CTL). The Commons is a showcase for Jefferson books and journals, peer-reviewed scholarly publications, unique historical collections from the University archives, and teaching tools. The Jefferson Digital Commons allows researchers and interested readers anywhere in the world to learn about and keep up to date with Jefferson scholarship. This article has been accepted for inclusion in Department of Psychiatry and Human Behavior Faculty Papers by an authorized administrator of the Jefferson Digital Commons. For more information, please contact: JeffersonDigitalCommons@jefferson.edu. 
Authors

Ashwin A. Patkar, Charles A. Marsden, Prakash C. Naik, David A. Kendall, Raman Gopalakrishnan, Michael J. Vergare, and Stephen P. Weinstein 


\section{Differences in Peripheral Noradrenergic Function among Actively Drinking and Abstinent Alcohol-dependent Individuals}

We examined whether excessive alcohol consumption was related to changes in plasma levels of noradrenaline (NA) and whether these changes recover following abstinence. We also explored whether there were differences in NA levels between Type I and Type II alcoholics and controls during active drinking and abstinence. Plasma concentrations of NA were determined in (1) 27 Caucasian men with alcohol dependence who were regularly drinking (active drinkers) within 24 hours of hospitalization, (2) 29 Caucasian alcohol-dependent men who were in remission (abstinent for a minimum of three months), and (3) 28 race- and gender-matched healthy controls. NA concentrations were significantly higher in actively drinking alcohol-dependent subjects compared to those in remission and controls. While Type I and Type II alcoholic individuals differed across clinical measures, NA levels were similar in the two subtypes. Both subtypes showed an elevation in NA levels during active drinking compared to controls, but NA levels did not differ between the two subtypes and controls during remission. The findings indicate that chronic exposure to alcohol may lead to disturbances in NA activity that may manifest in early abstinence. However, the changes in NA activity appears to normalize after a longer period of abstinence. Alterations in NA activity do not seem to be specific for Type I or Type II subtypes of alcoholism.

Several lines of evidence support the involvement of the central noradrenergic (NA) system in mediating the behavioral effects of alcohol (ethanol). For example, animal experiments have shown that acute alcohol exposure increases NA synthesis and release, leading to increased NA concentrations in the brain. $\underline{1}-\underline{3}$ The sedative, intoxicating, and locomotor effects of alcohol, as well as alcohol withdrawal, seem to be related at least in part to increased NA activity. $\underline{4}-\underline{6}$ Studies have also found that the central NA system may contribute to the rewarding effects of ethanol via its interaction with the dopaminergic pathways. 7 Further support for the role of NA in mediating the effects of alcohol comes from genetic knock-out experiments. Weinshenker et al. showed that mice lacking NA had a markedly reduced preference for ethanol and were hypersensitive to the sedative and hypothermic effects of ethanol.

Compared to the preclinical evidence supporting the effects of alcohol on NA function, there have been several inconsistencies in human data that have limited the interpretation of findings. The conflicting findings appear to be related to the pharmacokinetics of alcohol metabolism and several clinical variables that are difficult to control among humans. For example, alcohol's effects on NA seem to vary with dose, length of exposure, and stage of withdrawal. While a number of studies have replicated findings of increased NA activity during early withdrawal, $\underline{9}$, $\underline{12}$ data during intoxication and abstinence have been less consistent. While subsensitive alpha- 2 adrenergic receptor function has been reported in alcoholics following extended abstinence in some studies, 13 other studies could not replicate the findings. 14 Similarly, measurements of cerebrospinal fluid levels of monoamines in recently detoxified alcoholic patients 15 could not confirm previous reports of alterations in neuroendocrine responsivity of the NA system that were observed in similar patients.16 Moreover, the effects of acute ethanol administration have 
been often studied in healthy subjects $\underline{17}-19$ that bear little resemblance to clinical populations of alcoholic individuals.

Biological studies in alcohol dependence are further complicated by the heterogeneity of the clinical population.20 Studies of problem drinkers and their families have yielded two broad categories, termed Type I and Type II by Cloninger 21 and Type A and Type B by Babor et al.22 The Type II alcoholism is predominantly limited to men and represented by an earlier age of onset of alcohol problems, greater severity of problems, stronger familial and genetic influences, and higher impulsivity and violent behavior. In contrast, the Type I subgroup is more benign with a later onset, higher anxiety levels, slower developmental course, fewer complications, and a better prognosis. Biological studies have found the two subtypes may differ in measures of serotonin, $\underline{23}$ dopamine, 24 and monoamine oxidase activity. $\underline{25}$ Very few studies of the noradrenergic system during different stages of alcohol dependence have taken into account the distinct subgroups of alcoholic individuals.

In an earlier study that investigated serotonergic function in alcohol dependence, we found that Type II alcoholism was associated with increased platelet tritiated imipramine binding that persisted during abstinence.26 In the present study, that work is extended to examine the role of NA system in alcoholism. The primary objective was to examine the differences in peripheral NA levels in alcohol-dependent individuals during active drinking and abstinence. The secondary objective was to determine if the Type I and Type II subgroups of alcoholic patients differ from each other and from controls in measures of NA function.

\section{METHODS}

\section{Recruitment}

The sample comprised of three groups of male, Caucasian individuals:

- 1

27 alcohol-dependent inpatients who were still drinking.

- 2

29 alcohol-dependent subjects who were in remission (abstinent for a minimum of three months, termed "abstinent alcoholics").

- 3

28 healthy volunteers with no history of alcohol dependence or abuse, serving as the control population.

Over $90 \%$ of the clinical population from which the sample was drawn was of Caucasian background. We restricted the population to men because Type II alcoholism is predominately male-limited; also, significant variations of urinary NA have been reported in women.27 
The actively drinking alcohol-dependent subjects were recruited from a university-affiliated inpatient addiction unit in Nottingham, England. The inclusion criteria were:

- 1

$D S M-I V$ diagnosis of alcohol dependence. $\underline{28}$

- 2

No interruption of alcohol intake in the previous 72 hours and no clinical evidence of alcohol withdrawal.

- 3

Capable of giving informed consent.

Individuals with major depression, bipolar or psychotic disorders, serious medical disorders, or a positive urine drug screen (except benzodiazepines), as well as those receiving psychotropic medication (except benzodiazepines), were excluded from the study. Alcohol-dependent subjects were recruited, and blood samples were obtained within 24 hours of admission to the inpatient unit.

Abstinent alcoholics were recruited from a residential recovery house in Nottingham. Complete abstinence was mandated for residing in the recovery house, and attendance at Alcoholics Anonymous (AA) was encouraged. Most of the residents were in treatment at the outpatient clinic or day hospital affiliated with the addiction unit and underwent random breath tests. Subjects who were previously dependent on alcohol $(D S M-I V)$ but currently abstinent for at least three months were included in the study. The exclusion criteria were similar to those for the actively drinking group.

Controls were hospital employees or their relatives. They were excluded if they had a positive response to items on the CAGE questionnaire, had a positive breathalyzer reading, reported greater than 21 units of alcohol consumption per week in the previous month (one unit is equivalent to $10 \mathrm{ml}$ or $8 \mathrm{~g}$ of pure alcohol and corresponds to 4 fluid ounces of table wine, 1.5 fluid ounces of spirits, or 8 fluid ounces of beer), had a major psychiatric or medical disorder, or were receiving psychotropic medication.

\section{Behavioral and Clinical Assessments}

The study protocol was approved by the ethics committee of the hospital. After a description of the study, written informed consent was obtained. Alcohol-dependent individuals who volunteered for the study underwent a psychiatric interview and had their medical health assessed by medical history and physical examination. Alcohol history was recorded in a standardized format. Eligible subjects were asked to provide a urine sample and tested for breath alcohol levels. Subjects then completed a battery of psychological assessments that included the severity of alcohol dependence questionnaire (SADQ), 29 a visual analogue scale for craving 
(VAS), $\underline{30}$ and a questionnaire to classify subjects into Type I and Type II subtypes of alcoholism. $\underline{21}$

The SADQ is a 20-item, self-report questionnaire that assesses severity of alcohol dependence and requires about 20 minutes to complete. The items assess five functional areas: physical problems, emotional problems, pattern of drinking, amount of drinking, and withdrawal symptoms. For each item, the scores range from 0 (mild) to 3 (severe), so the total score can range from $0-60$. The reliability and validity of the instrument have been established in studies of alcoholic patients from United Kingdom. 29

The VAS has been frequently used to quantify craving for drugs and alcohol and has been used as a subjective measure of alcohol craving. 30 It can be completed in two minutes. Subjects were asked to rate their craving on a $100 \mathrm{~mm}$ horizontal line, and the distance from the left end of the line was measured in $\mathrm{mm}$ to provide an estimate of craving. The scores ranged from 0 (no craving) to 100.

The Type I/Type II classification was based on procedures outlined by Gilligan et al. 31 and Sullivan et al. .32 The questionnaire included 12 variables that were categorized as representing a Type I characteristic (eg, first dependence or abuse symptom after age 25) or a Type II characteristic (eg, family history of alcoholism). All positive responses to Type I items (ie, yes) were weighted positively (+) and all positive responses to Type II items were weighted negatively (-). A total score was obtained for each subject by summing scores for all items. Subjects with scores in the positive range were classified as Type I; individuals with negative scores received a Type II classification. A score of 0 was considered to represent "unclassified" grouping. The questionnaire required $10-15$ minutes to complete.

Consenting controls were screened using the CAGE questionnaire, 33 a clinical interview, a physical examination, and breath alcohol readings. The CAGE is a four-item questionnaire that takes about a minute to complete. It has been extensively employed as a screening tool to detect alcoholism in clinical settings. A positive response to one or more of the four questions has been found to be highly correlated with a diagnosis of alcoholism with a sensitivity of $84 \%$ and specificity of $90 \% . \underline{34}$

\section{The NA Assay}

Twenty $\mathrm{ml}$ of venous blood was collected from subjects and controls. For hospitalized, alcoholic subjects, blood was drawn within 24 hours of admission. The average time period from the time of admission to the time of blood collection was 7.4 hours. Blood samples were collected in the morning and transported to the laboratory on ice within two hours of blood draw to reduce the possibility of platelet aggregation. The samples were centrifuged within four hours of arrival at the laboratory to separate the plasma from the blood constituents. The plasma was separated and stored at $-80 \mathrm{C}$. In the laboratory, all samples were identified by code numbers and assayed as a single experiment to minimize variations in the plasma NA levels. The NA assay was performed using High Performance Liquid Chromatography (HPLC) with electrochemical detection optimized for the detection of NA. Details of the techniques for the NA assay have been previously described. $35-\underline{37}$ The protein assay was performed by the methods described by 
Lowry et al. $\underline{38}$ The NA concentrations were expressed in picomoles/mg of protein. The laboratory personnel were kept blind to the clinical data.

Blood samples from alcoholic subjects were also analyzed for serum Gamma Glutamyl Transpeptidase (GGT) and Mean Corpuscular Volume (MCV) values. Both elevated GGT and $\mathrm{MCV}$ are indicative of excessive alcohol consumption and reported to be elevated in $50-75 \%$ of alcoholic individuals. $\underline{39}$

\section{Statistical Analysis}

Analysis of variance (ANOVA) or independent t-tests (two-tailed) were employed to compare the three study groups and the Type I and Type II subgroups with respect to NA levels and scores on the clinical variables. Chi-square tests were used to compare dichotomous variables. Relationships between clinical variables and NA levels were examined using Pearson or point biserial correlations. When significant relationships were observed between NA levels and clinical measures, the data were reanalyzed using analyses of covariance (ANCOVA) to control for the effects of other dependent variables on NA.

\section{RESULTS}

\section{Subjects}

A total of 84 Caucasian men were studied: 27 were actively drinking, alcohol-dependent patients, 29 were alcohol-dependent individuals in remission (abstinent alcoholics), and 28 were healthy volunteers. Table 1 summarizes the demographic and clinical characteristics of the sample.

Table 1. Clinical Characteristics of Subjects and Controls

\begin{tabular}{|c|c|c|c|c|}
\hline Characteristics & $\begin{array}{l}\text { Actively drinking ( } n \\
=27)\end{array}$ & $\begin{array}{c}\text { Abstinent }(n= \\
\text { 29) }\end{array}$ & $\begin{array}{c}\text { Controls }(n= \\
\text { 28) }\end{array}$ & $\mathbf{F} / \mathbf{t} / \chi^{2}$ \\
\hline Age (years) & $39.4 \pm 10.1$ & $41.1 \pm 9.4$ & $36.6 \pm 6.4$ & $\mathrm{~F}=1.69$ \\
\hline Employed & $27.5 \%$ & $32.1 \%$ & $93.1 \%$ & $\begin{array}{l}\chi^{2}= \\
14.22 \dagger\end{array}$ \\
\hline Single & $68.9 \%$ & $75.0 \%$ & $44.8 \%$ & $\begin{array}{l}\chi^{2}= \\
8.26^{*}\end{array}$ \\
\hline $\begin{array}{l}\text { Duration of problem drinking } \\
\text { (years) }\end{array}$ & $19.2 \pm 10.4$ & $20.4 \pm 12.7$ & N/A & $\mathrm{t}=1.18$ \\
\hline $\begin{array}{l}\text { Severity of drinking (SADQ } \\
\text { scores) }\end{array}$ & $41.4 \pm 10.6$ & $46.5 \pm 9.9$ & N/A & $\mathrm{t}=1.32$ \\
\hline Craving (VAS scores) & $54.2 \pm 32.1$ & $29.6 \pm 19.7$ & N/A & $\mathrm{t}=2.50^{*}$ \\
\hline $\mathrm{MCV}+$ & $96 \pm 6$ & $91 \pm 9$ & N/A & $\mathrm{t}=1.38$ \\
\hline GGT § & $249 \pm 507$ & $48 \pm 42$ & N/A & $\mathrm{t}=3.61 \dagger$ \\
\hline Benzodiazepines II & $35.7 \%$ & $28.3 \%$ & N/A & $\chi^{2}=1.18$ \\
\hline
\end{tabular}




\section{Characteristics}

Tobacco smokers

\section{Actively drinking ( $n$ $=27$ )}

$89.6 \%$
29)

$92.8 \%$

\section{Controls $(n=$ \\ 28) \\ $\mathbf{F} / \mathbf{t} / \chi^{2}$ \\ $\chi^{2}=$ \\ $11.31 \dagger$}

- $* p<.05$, for $\mathrm{t}$ tests $\mathrm{df}=54$, for $\chi^{2} \mathrm{df}=1-2$.

- $\quad \dagger p<.01$, for $\mathrm{t}$ tests $\mathrm{df}=54$, for $\chi^{2} \mathrm{df}=1-2$.

- $\quad$ + Normal MCV values ranged from 76-97 fl.

- $\$$ Normal GGT values ranged from 10-50 IU/L.

- II Prescribed or present in the urine.

As seen in Table 1, the three groups did not differ significantly in age; however, alcoholdependent individuals were significantly more likely to be single, unemployed, and tobacco smokers than controls. While the actively drinking and abstinent patients did not differ in severity or duration of drinking, the former had higher craving scores and GGT levels than the latter group. Among patients who were actively drinking, the breath alcohol level was $121 \pm 84$ $\mathrm{mg}$, and two patients had alcoholic hepatitis. No individuals had tested positive for HIV; however, thirteen $(23.2 \%)$ alcoholic individuals had a history of medical diseases such as liver disease, asthma, hypertension, or diabetes, or were taking medications for their medical conditions. Five actively drinking subjects were taking benzodiazepines (BZ); however, ten $(35.7 \%)$ had evidence of recent use of BZ in urine. More than a quarter of abstinent individuals were prescribed BZ, and three were taking disulfiram. None of the alcoholics was prescribed more than $20 \mathrm{mg}$ of diazepam (or its equivalents) a day.

\section{Plasma NA Levels in Alcoholic Individuals, Abstinent Alcoholics, and Controls}

For technical reasons, four blood samples could not be analyzed. The NA data are reported for eighty individuals. The plasma NA levels significantly differed between actively drinking individuals $(5.08 \pm 2.32)$, abstinent patients $(3.15 \pm 1.94)$, and controls $(3.03 \pm 1.31)(\mathrm{F}[2,78]=$ $10.12, p<.001)$. Data analysis showed that NA levels were significantly higher in actively drinking compared to abstinent persons and controls; the latter two groups had similar NA concentrations. Figure 1 shows the distribution of plasma NA concentrations in the three groups. 


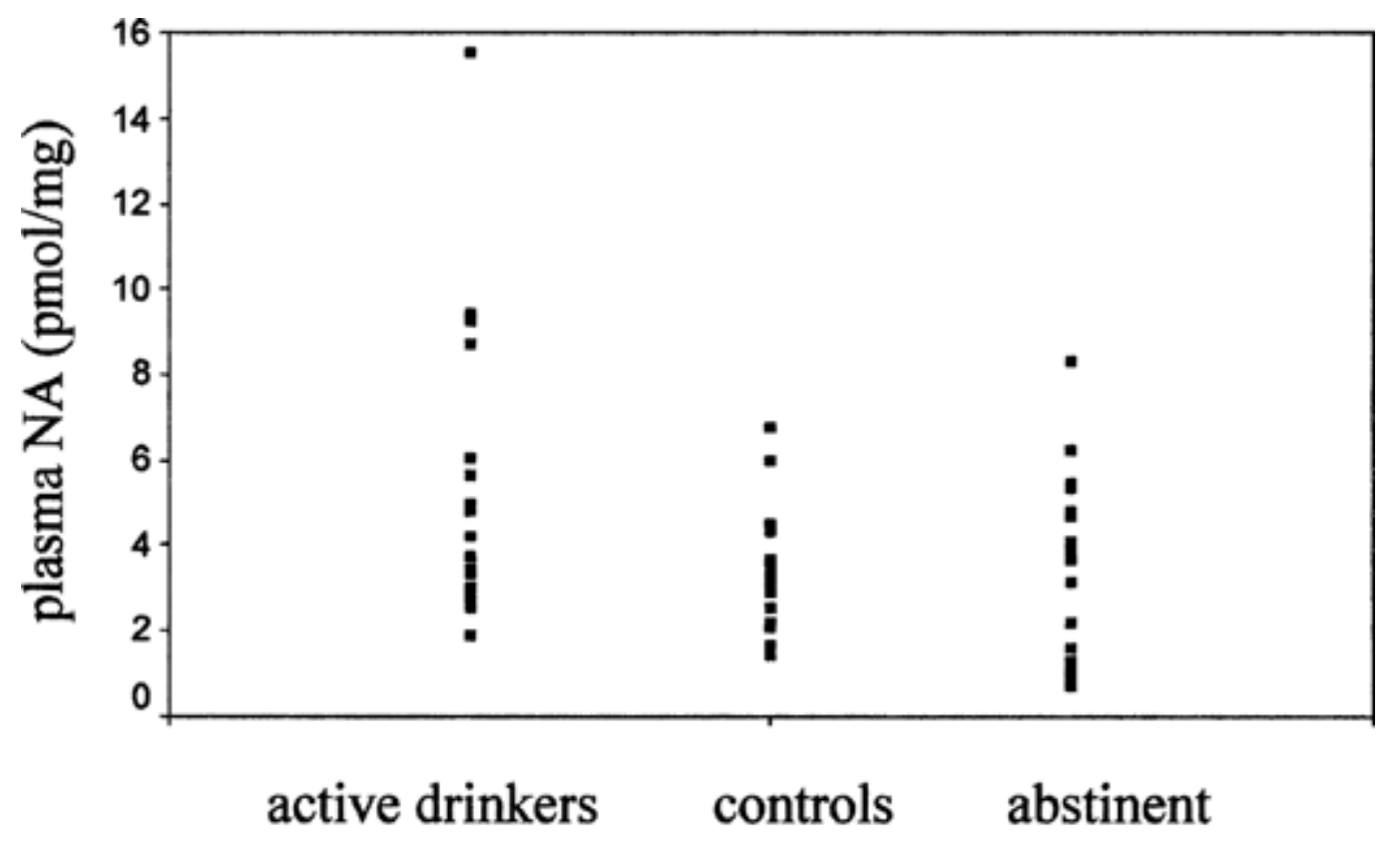

ANOVA: $\mathrm{F}(2,78)=10.12, p<.001$
Figure 1
Open in figure viewerPowerPoint
Plasma noradrenaline in active drinkers, controls, and abstinent alcoholics.

Since there were demographic differences between the three groups, we examined whether these variables were related to plasma NA levels. We found that NA levels were not significantly correlated with employment, marital status, or smoking status (X $\underline{2}$ ranged from 0.48 to $1.87, \mathrm{df}=$ $2, p>.05$ in each case), or with GGT values, breath alcohol levels, craving scores ( $\mathrm{r}$ ranged from .05 to $.17, p>.05$ in each case). We also compared NA levels between alcoholic individuals taking benzodiazepines and those who were not, and found no significant differences between the two groups $(\mathrm{X} \underline{2}=2.11, \mathrm{df}=1, p>.05)$. We then assessed the influence of comorbid medical diseases (eg, hypertension, asthma) on NA levels in alcoholic individuals by comparing the NA levels between patients with concurrent medical disorders $(n=13)$ and those without such disorders $(n=43)$. No significant difference was found between the two groups $=1.87, \mathrm{df}=1, p$ $>$.05).

We also examined the relationship between clinical variables (severity and duration of drinking, craving, and duration of abstinence) and plasma NA levels in alcoholic patients. A significant positive correlation was observed between NA levels and the total score $(\mathrm{r}=0.38, p<.05)$ and scores on the quantity $(\mathrm{r}=0.37, p<.05)$ and withdrawal sub-scales $(\mathrm{r}=.35, p<.05)$ of the SADQ. However, duration of drinking or craving scores were not significantly correlated with NA concentrations. Among abstinent alcoholics, the duration of abstinence was $5.9 \pm 3.3$ months. Two patients were abstinent more than twelve months, with the maximum duration being sixteen months. No significant correlation was observed between duration of abstinence and NA concentrations $(\mathrm{r}=.18, p>.05)$. 


\section{NA Levels in Type I and Type II Alcoholics}

In the combined alcoholic sample, 23 individuals were classified as Type I, 28 as Type II, and five were rated as "unclassified". While the two subtypes did not differ in demographics, Type II alcoholics were more likely to have a longer duration of drinking $(\mathrm{t}=2.58, \mathrm{df}=49, p<.05)$, higher GGT values $(\mathrm{t}=2.38, \mathrm{df}=49, p<.05)$, and higher scores on the physical problems subscale of the SADQ $(\mathrm{t}=2.17, \mathrm{df}=47, p<.05)$. The other clinical variables did not differ between the two subtypes. Because the alcohol-dependent group who were drinking regularly had higher NA levels than controls, we examined whether Type I $(n=12)$ and Type II $(n=13)$ individuals in this group differed in their NA levels. No significant differences in NA levels were observed between Type I $(4.96 \pm 1.98)$ and Type II $(4.98 \pm 2.73)$ individuals $(\mathrm{t}=.21, \mathrm{df}=23, p$ $>$.05). Since Type I and Type II individuals differed in certain SADQ measures and SADQ scores were related to NA levels, we reanalyzed the data covarying for SADQ scores. The ANCOVA analysis did not change the direction or significance of the findings.

\section{DISCUSSION}

\section{NA Levels During Alcohol Dependence and Abstinence}

The present study found that plasma NA levels were significantly elevated in chronic alcoholdependent men who were still drinking compared to those who were abstinent for at least three months and controls. Because blood samples in the alcohol-dependent group were drawn at different time points and breath alcohol levels were variable, it is difficult to uniformly characterize the clinical and physiological status of this group. Although some patients clinically had signs of intoxication and blood samples were collected before clinical signs of withdrawal were evident, patients had stopped drinking over seven hours before the blood draw. Therefore, it seems that physiologically most patients were in an early asymptomatic abstinent state. The increase in NA levels observed in this state is consistent with the animal data regarding the effects of alcohol on the noradrenergic system. $1, \underline{2}$ The results also support previous studies in humans that found plasma catecholamines to be increased following alcohol exposure and withdrawal $12, \underline{40}$ and reports of down-regulation of alpha-2 receptors in recently detoxified alcoholic subjects. $16, \underline{41}$ However, contrasting findings have also been reported possibly related to differences in stages of alcohol dependence. For example, Ibsen et al.42 have also reported normal NA concentrations in chronic, stable alcoholics, though it was unclear in this study whether subjects were in early withdrawal. Contrary to our expectations, breath alcohol levels were not correlated with plasma NA levels in the actively drinking group. One reason could be that a single breath alcohol value does not indicate whether blood alcohol levels were stable, increasing, or declining in a particular individual. It is likely that a proportion of patients might have reduced their consumption before hospitalization and, although intoxicated, may actually have had declining blood alcohol levels. Another reason for the lack of correlation between NA and alcohol levels could be individual differences in the magnitude of physiological tolerance and dependence on alcohol. We also failed to find a relationship between NA levels and craving during active drinking and abstinence, supporting findings from a recent study that did not observe a relationship between levels of NA metabolites in cerebrospinal fluid cue-induced craving in alcohol-dependent subjects. 15 It seems that dopaminergic and serotonergic 
mechanisms may be preferentially involved in mediating craving for drugs and alcohol along with cognitive, affective, and psychological factors. $\underline{43}, \underline{44}$

\section{NA Levels in Type I and Type II Alcoholism}

As expected, the Type II alcoholics had a longer duration of drinking, more physical problems, and higher levels of GGT and also tended to drink more compared to the Type I group. After correcting for these clinical differences, NA levels were similar in Type I and type II alcoholics during active drinking and abstinence. While there is a large body of evidence that Type I and Type II alcoholics may differ in serotonergic $\underline{23}, \underline{45}$ and dopaminergic $\underline{24}$ function the data on differences in NA function between the two subtypes have been less robust. While early research indicated that there may be differences in platelet mono-amine oxidase (MAO) activity between the two subtypes $\underline{21}, \underline{25}$ two genetic studies have yielded negative results. $\underline{46}, \underline{47}$ Although there have been suggestions that the Type I alcoholics may have higher anxiety levels than the Type II subgroup our study did not find any differences in plasma NA between the two subtypes. However it is possible that although NA levels may have normalized within a few weeks of abstinence changes in adrenergic receptor function could persist for much longer periods. 13 Further research comparing adrenergic receptor function between the two subtypes during abstinence may clarify whether the two subtypes are characterized by differences in the noradrenergic system.

\section{Methodological Issues}

Given the variations in effects of alcohol on the NA metabolism selection of sample becomes critical in biological studies of alcoholics. Because our objective was to compare chronic alcohol-dependent patients who were actively drinking with those who were abstinent we studied alcoholics before clinical withdrawal was evident and recruited subjects with elevated levels of alcohol in the breath. This also ensured that subjects were not started on detoxification regimens with large doses of benzodiazepines that can suppress NA activity. $\underline{48}$ Similarly abstinence was determined by objective data controls were clinically screened and Type I and Type II classifications were obtained using standardized assessments. We also examined the effects of potential confounding variables such as duration and severity of drinking and the use of tobacco and concomitant benzodiazepines. Nevertheless the data have to be interpreted in light of certain limitations. First the stress of hospitalization itself may have increased the levels of NA in the actively drinking group. Because we did not measure other biochemical indices such as cortisol levels or NA metabolites it was not possible to distinguish between the effects of alcohol and stress of hospitalization in our subjects. However studies have shown that stress by itself cannot explain the increases in NA seen in hospitalized alcoholics. $\underline{49}$ Also it is likely that both controls - who were unaccustomed to screening interviews, breath tests, and venepuncture — and recovering alcoholics - whose behavior was heavily regulated in the residential facilities - also experienced stress. Finally, the interaction between alcohol and stress is not necessarily synergistic but may also be antagonistic. For example, in experimental animals, alcohol given prior to immobilization (ie, stress) has been found to reduce the restraint-induced elevations in NA. 50 Given these circumstances, it seems that the over $60 \%$ increase in NA in the actively drinking alcoholics compared to the other two groups does not seem to be due to stress of hospitalization alone. Second, the extent to which plasma NA reflects changes in NA function in 
human brain is unclear. Certain metabolic differences are present between periphery and the brain in humans that may need to be considered during interpretation of data (eg, high-affinity alcohol dehydrogenase enzyme is absent in the brain 51). However, human studies have found correlations between brain and plasma levels of MHPG and NA, and plasma NA and its metabolites are believed to be a major determinant of levels of NA in the cerebrospinal fluid. $\underline{52}$ We also acknowledge that the small sample size may have affected the ability to detect hypothesized differences in NA levels between the Type I and Type II subtypes or the possible differences between alcoholics with high and low amounts of drinking. Finally, since the sample was restricted to Caucasian men to control for possible race and gender effects, the data cannot be generalized to women and to individuals from other ethnic backgrounds.

\section{Clinical Significance and Conclusions}

The increases in NA levels in alcohol-dependent individuals who were actively drinking indicate that chronic exposure to alcohol may lead to disturbances in NA activity that may be manifested in early abstinence. The normalization of NA levels with a longer period of abstinence suggests that the alcohol-induced NA changes may be state-dependent phenomena. Although the Type I and Type II alcoholics differ clinically, NA measures do not appear to distinguish between the two subtypes. Determining the influence of selective NA agents on the central effects of alcohol as well as examining the relationships between NA and dopaminergic mechanisms during different stages of alcohol dependence may clarify the importance of the NA system in mediating the addictive properties of alcohol in humans.

This research was supported by the Trent Regional Health Authority and Upjohn-Duphar Pharmaceuticals. The work was carried out at the Drug and Alcohol Center, Mapperley Hospital, and Department of Physiology and Pharmacology, University of Nottingham Medical School, Nottingham, UK. Drs. Patkar and Naik were formerly associated with the Drug and Alcohol Center, Maperley Hospital.

We thank Dr Philip McLean for medical input and David Forster for technical assistance.

\section{REFERENCES}

- 1 Engel, CK, Rudberg, U. Age-dependent effects of ethanol on central monoamine synthesis in the male rat. Acta Pharmacol Toxicol. 1985; 57: 336- 339.

- 2 Rabin, RA, Wolfe, BB, Dibner, MK, et al. Effects of ethanol administration and withdrawal on neurotransmitter receptor systems inC57 mice. J Pharmacol Exp Ther. 1980; 213: 491- 496.

- 3 Thiele, TE, Cubero, I, Dijk, G, et al. Ethanol-induced c-fos expression in catecholamine-and neuropeptide Y-producing neurons in rat brainstem. Alcohol Clin Exp Res. 2000; 24: 802- 809.Noradrenergic Function in Alcohol Dependence. 
- 4 Hodge, CW, Slawecki, CJ, Aiken, AS. Norepi-nephrine and serotonin receptors in the periventricular nucleus interactively modulate ethanol consumption. Alcohol Clin Exp Res. 1996; 20: 1669- 1674.

- 5 Le, AD, Kiianmaa, K, Cunningham, CL, et al. Neurobiological processes in alcohol addiction. Alcohol Clin Exp Res. 2001; 25( 5 Suppl ISBRA): 144S-151S.

- 6 Wang, Y, Freund, RK, Palmer, MR. Potentiation of ethanol effects in cerebellum by activation of endogenous noradrenergic inputs. J Pharmacol Exp Ther. 1999; 288: 211220.

- 7 Adell, A, Myers, RD. 5-HT, dopamine, norepinephrine, and related metabolites in brain of low alcohol drinking (LAD) rats shift after chronic intra-hippocampal infusion of Harman. Neurochem Res. 1995; 20: 209- 215.

- 8 Weinshenker, D, Rust, NC, Miller, NS, et al. Ethanol-associated behaviors of mice lacking norepinephrine. J Neurosci. 2000; 20: 3157- 3164.

- 9 Balldin, J, Berggren, U, Engel, J, et al. Alpha-2-adrenoceptor sensitivity in early alcohol withdrawal. Biol Psychiatry. 1992; 31: 712- 719.

- 10 Linnoila, M. Alcohol withdrawal and noradrenergic function. Ann Intern Med. 1987; 107: 875- 889.

- 11 Nutt, D, Glue, P, Molyneux, S, et al. Alpha-2-adrenoceptor function in alcohol withdrawal: a pilot study of the effects of i.v. clonidine in alcoholics and normals. Alcohol Clin Exp Res. 1988; 12: 14- 18.

- 12 Smith, AJ, Brent, PJ, Henry, DA, et al. Plasma noradrenaline, platelet a2adrenoceptors, and functional scores during ethanol withdrawal. Alcohol Clin Exp Res. 1990; 14: 497- 502.

- 13 Berggren, U, Fahlke, C, Norrby, A, et al. Subsensitive alpha-2 adrenoceptor function in male alcohol-dependent individuals during 6 months of abstinence. Drug Alcohol Depend 2000; 57: 255- 260.

- 14 Muller, N, Hoehe, M, Klein, HE, et al. Endocrinological studies in alcoholics during withdrawal and after abstinence. Psychoneuroendocrinology. 1989; 14: 113- 123.

- 15 Petrakis, IL, Trevisan, L, D'Souza, C, et al. CSF monoamine metabolite and beta endorphin levels in recently detoxified alcoholics and healthy controls. Alcohol Clin Exp Res. 1999; 23: 1336- 1341.

- 16 Krystal, JH, Webb, E, Cooney, NL, et al. Serotonergic and noradrenergic dysregulation in alcoholism: m-chlorophenylpiperazine and yohimbene effects in recently detoxified alcoholics and healthy comparison subjects. Am J Psychiatry. 1996; 153: 8392.

- 17 Howes, LG, Reid, JL. Changes in plasma free 3,4-dihydroxyphenylethylene glycol and noradrena-line levels after acute alcohol administration. Clin Sci. 1985; 69: 423- 428.

- 18 Ireland, M, Vandongen, R, Davidson, L, et al. Acute effects of moderate alcohol consumption on blood pressure and plasma catecholamines. Clin Sci. 1984; 66: 643- 648.

- 19 McDougle, CJ, Krystal, JH, Price, LH, et al. Noradrenergic response to acute ethanol administration in healthy subjects: comparison with intravenous yohimbene. Psychopharmacology. 1995; 118: 127- 135.

- 20 Carpenter, KM, Hasin, DS. Reliability and discriminant validity of the Type I/II and Type A/B alcoholic subtype classifications in untreated problem drinkers: a test of the Apollonian-Dionysian hypothesis. Drug Alcohol Depend. 2001; 63: 51 - 67. 
- 21 Cloninger, CR. Neurogenetic adaptive mechanisms in alcoholism. Science. 1987; 236: 410- 416.

- 22 Babor, TF, Dolinsky, ZS, Meyer, MH, et al. Types of alcoholics: concurrent and predictive validity of some classification schemes. Br. J. Addic. 1992; 87( 10): 14151431.

- 23 George, DT, Benkelfat, C, Rawlings, RR, et al. Behavioral and neuroendocrine responses to $\mathrm{m}$-chlorophenylpiperazine in subtypes of alcoholics and in healthy comparison subjects. Am J Psychiatry. 1997; 154: 81- 87.

- 24 Tupala, E, Kuidda, JT, Hall, H, et al. Measurement of the striatal dopamine transporter density and heterogeneity in type 1 alcoholics using human whole hemisphere autoradiography. Neuroimage. 2001; 14: 87- 94.

- 25 Knorring, L, Oreland, L, Knorring, AL. Personality traits and platelet MAO activity in alcohol and drug abusing teenage boys. Acta Psychiatry Scand. 1987; 75: 307- 314.

- 26 Patkar, AA, Naik, P, Al-Chalabi, T, et al. Platelet (3)H Imipramine binding in type I and type II alcoholism. Human Psychopharmacology. 1995; 10: 1- 6.

- 27 Hansen, AM, Garde, AH, Skovgaard, LT, et al. Seasonal and biological variation of urinary epinephrine, norepinephrine, and cortisol in healthy women. Clin Chim Acta. 2001; 309: 25- 35.

- 28 American Psychiatric Association. Diagnostic and Statistical Manual of Mental Disorders. 4th ed. Washington, DC: American Psychiatric Press; 1994.

- 29 Stockwell, T, Murphy, D, Hodgson, R. The severity of alcohol dependence questionnaire: its use, reliability and validity. British Journal of Addiction. 1983; 78: 145156.

- 30 McCormack, HM, Horne, DJ, Sheather, S. Clinical applications of visual analog scales: a critical review. Psychol Med. 1988; 18: 1007- 1019.

- 31 Gilligan, SB, Reich, T, Cloninger, RC. Alcohol related symptoms in heterogeneous families of hospitalized alcoholics. Alcohol Clin Exp Res. 1988; 12: 671- 678.

- 32 Sullivan, JL, Baeziger, JC, Wagner, DL, et al. Plate-let MAO in subtypes of alcoholism. Biol. Psychiatry. 1990; 27: 911- 922.

- 33 Ewing, JA. Detecting alcoholism: the CAGE questionnaire. JAMA. 1984; 252: 19051907.

- 34 Soderstrom, CA, Smith, GS, Kufera, JA, et al. The accuracy of the CAGE, the Brief Michigan Alcoholism Screening Test, and the Alcohol Use Disorders Identification Test in screening trauma center patients for alcoholism. J Trauma. 1997; 43: 962- 969.

- 35 Foster, CD, Taylor, JY, Macdonald, IA. The assay of the catecholamine content of small volumes of human plasma. In: H Rollema, BHC Westerink, WJ Drijfhout, eds. Monitoring Molecules in Neuroscience. Groningen: University Center for Pharmacy; 1991: 173- 175.

- 36 Fulford, AJ, Marsden, CA. Effect of isolation-rearing on noradrenaline release in rat hypothalamus and hippocampus in vitro. Brain Res. 1997; 748: 93- 99.

- 37 Lapiz, MD, Mateo, Y, Durkin, S, et al. Effects of central noradrenaline depletion by the selective neurotoxin DSp-4 on the behavior of the isolated rat in the elevated maze plus maze and water maze. Psychopharmacology(Berl). 2001; 155: 251- 259.

- 38 Lowry, O, Rosebrough, NJ, Farr, AL, et al. Protein measurement with the folin phenol reagent. J Biol Chem. 1951; 193: 265- 275. 
- 39 Allemann, PI. Markers for excessive alcohol use (screening). Ther Umsch. 2000; 57: 185- 190.

- 40 Maki, T, Toivonen, L, Koskinen, P, et al. Effect of ethanol drinking, hangover, and exercise on adrenergic activity and heart rate variability in patients with a history of alcohol-induced atrial fibrillation. Am J Cardiol. 1998; 82: 317- 322.

- 41 Fahlke, C, Berggren, U, Lundborg, C, Balldin, J. Psychopathology in alcohol withdrawal: relationship to alpha 2- adrenoceptor function. Alcohol Alcohol. 1999; 34: 750- 759 .

- 42 Ibsen, H, Christensen, MJ, Rasmussen, S, et al. The influence of chronic high alcohol intakeon blood pressure, plasma noradrenaline concentration and plasma renin concentration. Clin Sci. 1981; 61: 377- 379.

- 43 Schneider, F, Habel, U, Wagner, M, et al. Subcortical correlates of craving in recently abstinent alcoholic patients. Am J Psychiatry. 2001; 158: 1075- 1083.

- 44 Patkar, AA, Naik, P, Zaman, K, et al. Plasma serotonin (5-HT) levels and craving in alcohol dependent subjects during withdrawal [abstract]. Journal of Psychopharmacology. 1994; A34: 133.

- 45 Roy, A, Virkkunen, M, Linnoila, M. Reduced central turnover in a subgroup of alcoholics? Progresses in Neuropharmacology and Biological Psychiatry. 1987; 11: 173177.

- 46 Parsian, A, Suarez, BK, Tabakoff, B, et al. Mono-amine oxidases and alcoholism: I. Studies in unrelated alcoholics and normal controls. Am J Med Genet. 1995; 60: 409416.

- 47 Suarez, BK, Hampe, CL, Parsian, A, et al. Mono-amine oxidases and alcoholism: II. Studies in alcoholic families. Am J Med Genet. 1995; 60: 417- 423.

- 48 Finlay, JM, Zigmond, MJ, Abercombie, ED. Increased dopamine and norpepinephrine release in medial prefrontal cortex induced by acute and chronic stress: effects of diazepam. Neuroscience. 1995; 64: 619- 628.

- 49 Stanford, SC, Mikhail, G, Salmon, P, et al. Psychological stress does not affect plasma catecholamines in subjects with cardiovascular disorder. Pharmacol Biochem Behav. 1997; 58: 1167- 1174.

- 50 Patel, VA, Pohorecky, LA. Interaction of stress and ethanol: effect on B-endorphin and catecholamines. Alcohol Clin Exp Res. 1988; 12: 785- 788.

- 51 Linnoila, M, Guthrie, S, Lane, EA, et al. Clinical studies on norepinephrine metabolism: how to interpret the numbers. Psychiatry Res. 1986; 17: 229- 239.

- 52 Kopin, IJ, Gordon, EK, Jimerson, DC, et al. Relationship between plasma and cerebrospinal fluid levels of 3- methoxy-4-hydroxyphenyl glycol. Science. 1983; 219: 7375. 